\title{
HOSPITALIDAD Digital \\ Un concepto para la educación del siglo XXI
}

Digital Hospitality: a Concept for the Education of the 21st Century

Juan Alejandro Henriquez Peñailillo

Universidad de las Américas, Chile

\begin{tabular}{|c|c|}
\hline KEY WORDS & ABSTRACT \\
\hline Digital hospitality & Digital hospitality was born as a reflective approach from hermeneutics, \\
\hline Digital divide & presenting itself as a proposal for an advanced digital era, but which has \\
\hline Human rights & not yet managed to break the barriers of inequality, evidenced by the still \\
\hline Digital illiteracy & existing digital divide. We present this definition from an ethical \\
\hline Hermeneutics & reflection, respectful of diversity and with a human rights approach. We \\
\hline Digital education & hope that digital hospitality can be incorporated from the pre-school to \\
\hline Translation & $\begin{array}{l}\text { the tertiary system, where teachers develop this competence that favors } \\
\text { educational innovation and that seeks to reduce the gap and digital } \\
\text { illiteracy present in the 21st century. }\end{array}$ \\
\hline
\end{tabular}

\section{PALABRAS CLAVE}

Hospitalidad digital

Brecha digital

Derechos humanos

Alfabetización digital

Hermenéutica

Educación digital

Traducción

\section{RESUMEN}

La hospitalidad digital nace como una aproximación reflexiva desde la hermenéutica, presentándose como una propuesta para una avanzada era digital, pero que no logra aún romper las barreras propias de la desigualdad, evidenciada en la aún existente brecha digital. Presentamos esta definición desde una reflexión ética, respetuosa de la diversidad y con enfoque de derechos humanos. La hospitalidad digital, esperamos pueda incorporarse desde el sistema pre-escolar al terciario, donde los y las docentes desarrollen esta competencia que favorece la innovación educativa y que busca reducir la brecha y el analfabetismo digital presentes en el siglo XXI. 


\section{Hacia un concepto de hospitalidad digital desde la hermenéutica}

$\mathrm{L}$

a existencia de una determinante brecha digital y un bajo nivel de alfabetización digital tanto en docentes como en sus estudiantes, al menos en entornos digitales educativos y en lo referido a una competencia comunicativa multimodal, como indica Cassany (2002), nos lleva a proponer este artículo donde queremos proponer, además, una lectura vinculante entre los conceptos de hospitalidad y traducción, a la luz de lo planteado por nuestro autor principal, Paul Ricoeur (2005). Esto con el fin de cimentar una definición que proponemos para enfrentar dicha brecha digital (Rodríguez, 2006) presente en la creciente era digital (Pérez, 2013), aún en el siglo XXI y agravada aún más por la pandemia. Nos referimos al concepto de hospitalidad digital desde una perspectiva ética y educativa.

Para el desarrollo de este artículo, se empleará como metodología aquella basada en un paradigma cualitativo, conformando una investigación sustentada en la fenomenología hermenéutica bajo el método de explicacióncomprensión, donde su autor afirma que:

La comprensión es más bien el momento no metodológico que, en las ciencias de la interpretación, se combina con el momento metodológico de la explicación. Este momento precede, acompaña, clausura y así envuelve a la explicación. A su vez, la explicación desarrolla analíticamente la comprensión. (Ricoeur, 1999).

La hermenéutica filosófica, pensada desde una fenomenología hermenéutica permite la vinculación entre diversas teorías filosóficas y la praxis fáctica. Además, en ella "se impone la interpretación como tarea fundamental de la filosofía" (Ferraris, 2005). Esta aclaración nos permite un distanciamiento importante sobre la posibilidad de entender a la hermenéutica filosófica sólo como una interpretación de textos filosóficos. Es así como nos encontramos con vinculaciones de la hermenéutica con el expresar (afirmar, hablar), explicar (interpretar, aclarar) y traducir (trasladar). Un aporte sumamente interesante, es el dado por Grondin (1999) al indicarnos que las tres direcciones mencionadas respecto al sentido etimológico de hermenéutica pueden sintetizarse en un solo concepto: comprensión.

El género escogido para desarrollar la investigación bajo esta metodología es el del ensayo (Cervera, 2005).

Comenzaremos con categorizar hermenéuticamente las relaciones posibles entre lo que el autor entendía por hospitalidad (Ricoeur, 2005) y lo que aporta a nuestra propuesta y reflexión sobre una ética de la hospitalidad en contexto de un amplio desarrollo de tecnologías digitales al servicio de la educación y la forma de comprender el acto de traducir que, según expondremos, supera con creces el ámbito de lo lingüístico.

Por ello decimos que el concepto entra en el campo de lo ético, ya que:

La hermenéutica en la era digital tiene un carácter eminentemente ético, es decir que de ella emana la ética de la información, la cual se propone observar y problematizar el uso de la diferencia entre el debilitamiento o el endurecimiento de las estructuras digitales no sólo en vistas a la posible y real explotación, control y manipulación de las sociedades sino también en el horizonte más amplio del impacto de la técnica digital en todo tipo de procesos y productos naturales y artificiales así como en la hibridación de la técnica con la naturaleza y el mundo humano. (Capurro, 2010)

Respecto al concepto de traducción podemos decir lo siguiente.

Díez Fischer (2010), haciendo lectura de Ricoeur, señala primero, la imposibilidad misma de traducir, dada la importante diversidad de lenguas que muestra una heterogeneidad radical. Segundo, la existencia de un fondo común entre las lenguas ya sea pensando en la existencia de una lengua originaria o de una lengua universal. Como sea, "la traducción nos enfrenta [...] a una catástrofe lingüística inevitable" dice.

$\mathrm{Si}$ comprender es traducir, entonces deducimos que la catástrofe se da también en el encuentro diario con otro u otra que nos está interpretando constantemente. $\mathrm{Y}$, a pesar de todas las dificultades existentes para traducir, es innegable el factum lingüístico de que traducimos. Al hablar de traducción, Ricoeur (2005) delimita el terreno desde el cual habla. 
Bajo las condiciones de este terreno nos muestra que la traducción es siempre un constante desafío, con dificultades e imposibilidades. Nos indica que dichas dificultades aluden al término francés épreuve, en tanto "pena experimentada" y en tanto "prueba". Este concepto de traducción es relevante para nuestro propósito, dado que la era digital nos obliga permanentemente a comprender lo propio de lo extranjero, como explicaremos más adelante, que en este caso es el lenguaje propio de los entornos virtuales y tecnologías de la información y comunicación, lo cual se transforma en lo que debemos traducir.

El traductor queda como mediador entre el lector y el texto, no cualquier texto, el texto "extranjero". Aquí es donde entra la comprensión de la hospitalidad como un ejercicio de traducción de otro que es extranjero ante el sí mismo. Es por ello por lo que asumiremos el acto de traducir, más allá de los límites propios de la diversidad de idiomas. Entendemos el escenario de Babel, pero también el encuentro cotidiano de las personas, en ese entramado fáctico que desplaza el entender al extranjero (lingüísticamente hablando) sólo como quien procede de otra nación o país, es también aquel otro $u$ otra que nos interpela en una conversación o en un encuentro gestual y en un contexto de brecha digital nos referimos a la traducción intergeneracional, a la traducción de modelos educativos, de entornos de enseñanzaaprendizaje, entre otros.

Así pues, el acto de traducir deja al traductor como mediador de aquel encuentro. Ricoeur (2005) propone que esta relación permite el vínculo que él hace al comparar la "tarea del traductor" con el "trabajo del recuerdo" y el "trabajo del duelo". Respecto al trabajo del recuerdo, el autor plantea que el traductor encuentra de diversos modos una resistencia a su labor; presunción de no traducibilidad (fantasma de traducción perfecta), zonas de intraducibilidad. Esto genera una insatisfacción por su obra.

Respecto al trabajo del duelo, Ricoeur (2005) es claro, lo resume en la idea de "renunciar al ideal de la traducción perfecta". Al reflexionar sobre esta dificultad, encontramos atisbos de una oportunidad también, ya que permite al traductor, pensar-se ya no desde la imposibilidad de su traducir. Para efecto de nuestro artículo, esto es clave al momento de colocar nuestra atención, a modo de ejemplo, en la actual brecha digital con el foco de la diferencia generacional, ya que dicho trabajo del duelo se puede traducir como la renuncia de los docentes de avanzada edad hacia las nuevas tecnologías. Ahora bien, claro está que esto no está determinado sólo por un factor etario, especialmente cuando hablamos del uso de tecnologías de la información y conocimiento (TIC) en las aulas, ya que también existen factores de índole organizacional, económico $\mathrm{y}$, hasta, sanitarios, como bien ha reflejado la pandemia.

Explica, además, el deseo de la traducción perfecta desde el ejemplo de la omnitraducción y el del lenguaje puro. Esto nos permite abordar la necesidad de asumir la tarea del traductor desde el duelo, ya que permite, según Ricoeur, la felicidad de traducir, "Y es ese duelo de la traducción absoluta lo que va de la mano de la felicidad de traducir" (Ricoeur, 2005). Aquí entramos en lo que Ricoeur, llama hospitalidad lingüística. Hospedar la palabra del extranjero.

Ricoeur (2005), permite pensar el problema de la hospitalidad como una posibilidad cierta de encuentro, desde el desencuentro. ¿Y si la traducción fuese comprensión?, es más, recuerda que según Steiner (1977) "comprender es traducir". No es acaso posible intuir que de una hospitalidad lingüística podemos llegar a una ética de la hospitalidad. El texto por traducir sería la expresión de una otredad, pero otro u otra con rostro, con identidad, con historia. A diario vivimos el encuentro con otras personas, donde muchas veces nos angustiamos por no poder traducir perfectamente el mensaje de sus palabras o de sus gestos, si ese ideal de traducción perfecta lo asumimos desde el trabajo del duelo, podríamos buscar la comprensión del otro sin quedarnos fijos en la imposibilidad.

Así Ricoeur alienta a hospedar, más allá de la palabra del extranjero, al extranjero mismo. Como Ricoeur (2005) plantea desde Hölderlin: "lo que es propio debe aprenderse tan bien como lo extranjero".

Hemos pasado rápidamente desde una mirada conceptual de hospitalidad a una de hospitalidad lingüística, paso donde la traducción juega un rol preponderante. Por lo 
mismo, entendemos que el traducir es realmente una tarea $\mathrm{y}$, en el plano de una ética de la hospitalidad, puede entenderse como la tarea más importante para dar cuenta del encuentro con otro u otra que nos interpela e interpreta constantemente. Siguiendo esta línea argumentativa no podemos soslayar la posibilidad de entender la traducción como una capacidad del ser humano, somo capaces de traducir. Esta traducción toca profundamente la brecha digital y su indeseado efecto del analfabetismo digital, ya que entre los que tienen acceso a la información y los que no, sólo queda el acto de traducir contextos (también virtuales).

\section{Acepciones etimológicas del concepto hospitalidad}

Para fines de este artículo, entendemos que el concepto de hospitalidad corresponde a las acepciones latinas hospes y hostis, dado que ambas palabras nos permiten vislumbrar la posibilidad de la acogida desde el ipse, en tanto sí-mismo. Si bien es cierto, el principal autor que trabajamos en este apartado, Paul Ricoeur, no alude a la era digital y su respectivo contexto y especificidad, sí tomaremos sus lecturas y conceptos para dar cuenta del marco teórico que sustentará la propuesta específica de un nuevo concepto, hospitalidad digital.

En innumerables ocasiones, nos encontramos con personas y contextos que son huésped y enemigo al mismo tiempo. Esta situación nos coloca en la reflexión de pensar el sí mismo desde un dualismo existencial, por ser ipse y otro. Propongo esta categoría dual desde lo planteado por Ricoeur en "Ser y tiempo" (1996), en tanto síntesis de su pensamiento, al decir que "sí mismo como otro sugiere, en principio, que la ipseidad del sí mismo implica la alteridad en un grado tan íntimo que no se puede pensar en una sin la otra." El uso de internet y las redes sociales nos muestra con claridad esta idea de cruce entre el huésped y enemigo, dada la gran cantidad de comunidades virtuales que se crean y luego se separan en virtud de que su comunicación responde a un tiempo limitado, según dure la concreción de sus objetivos, como es el caso de las comunidades virtuales de aprendizaje (Cabero y Llorente, 2010).
Lo anterior, también es fundamentado desde el análisis sociolingüístico de Benveniste (1983), quien permitió ofrecer matices al concepto de 'enemigo' (hostis), ya que éste daría cuenta de algo contrario a la posibilidad misma de acogida de otro. Así es que da cuenta, también, que de hostis se deriva un significado cercano al del extranjero no romano que se iguala en derechos a él.

Un hostis no es un extranjero en general. A diferencia del peregrinus que habita fuera de los límites del territorio, hostis es 'el extranjero, en tanto que se le reconocen derechos iguales que a los ciudadanos romanos'. Este reconocimiento de derechos implica una cierta relación de reciprocidad, supone una convención: no se dice hostis a cualquiera que no sea romano. Un vínculo de igualdad y de reciprocidad se establece entre este extranjero y el ciudadano de Roma [...] hostis significa 'aquel que está en relaciones de compensación'; lo que es el fundamento de la institución de la hospitalidad. (Benveniste, 1983. 93).

Es importante destacar la noción de igualdad de derechos en este caso, ya que nos permitirá indagar en claves éticas y de un enfoque de derechos humanos.

Hablar de la hospitalidad digital desde un enfoque de derecho, nos permite reflexionar sobre la importancia de reconocernos en igualdad de oportunidades de acceso a la tecnología digital, por ejemplo. En el libro Brecha digital y sus determinantes, el autor cita a Kofi Annan:

Mucha gente carece de muchas cosas como, por ejemplo: trabajo, habitación, alimentación, cuidados de salud y agua potable. Si hoy los privamos del acceso a los servicios de telecomunicaciones básicas esto es tan grave como las otras privaciones y en realidad reduce las oportunidades para encontrarles solución. (Rodríguez, 2006, 2)

Podemos afirmar que, para lograr una real comprensión del otro, necesitamos entendernos desde una comprensión del sí mismo. Y no sólo una comprensión del sí mismo es necesaria, sino también una "estima de sí mismo". (Ricoeur, 1993) Según el autor, esta estima no alude a un tipo de egoísmo o individualismo, sino por el contrario, sugiere un encuentro reflexivo con un 
otro. Tal es el caso de internet, donde se juega el binomio de individuo y comunidad.

Este elemento, el de la estima, es para Ricoeur (1993), una parte de la cuarta capa fenomenológica, la capa de la vida ética, algo muy gráfico para nuestros intereses, por cierto. En esta capa, es la categoría de la responsabilidad la que vuelve ético el elemento de la estima. Y si llevamos esto al plano de la educación, sin duda nos encontramos con un rol docente que desde su responsabilidad para con sus estudiantes, desenvuelve una relación de estima que debe considerar un nivel de reconocimiento del otro a tal grado, que le permita favorecer aprendizajes significativos y con sentido, atendiendo a las nuevas formas de enseñar/aprender y al contexto tecnológico digital en el que nos situamos.

En este punto entran las llamadas eemociones, dado que el tema de la estima requiere especial atención al momento de configurar y diseñar espacios virtuales de aprendizaje. Es así como la perspectiva científica de las emociones se sustenta en las siguientes consideraciones: - Cuando hablamos de sentimientos no nos
referimos a un proceso exclusivamente
individual e interno, sino a un proceso
relacional. La emoción se construye
socialmente.

- Las emociones están mediadas por instrumentos y recursos culturales de naturaleza simbólica que nos proveen los contextos sociales. Estos instrumentos son clave para construir nuestra identidad (autoestima, estilo afectivo, etc.).

- Los sentimientos tienen un horizonte moral, es decir, suponen indicadores de la relación que establecemos con los contextos en tanto que éstos encarnan unos determinados valores culturales y actuamos en relación con ellos privilegiándolos o rechazándolos. (Rebollo, García, Barragán, Buzón y Vega, 2008, 4)

Sobre lo anterior, me detengo en un tipo de estima y de acogida, que aportará también al concepto de hospitalidad digital, me refiero al concepto de comprensión, del cual es posible señalar que uno se prende del otro, se sujeta del otro, que, en tanto sujeto a comprender, también se prende de uno. Ese prender no es más que el mismo implicarse con el otro, aproximarse al otro en tanto prójimo y sujeto de derecho, por ejemplo.

La idea de hospitalidad es inseparable de la idea de prójimo: aquel que está próximo, pero en el sentido de que su vida me concierne. Por eso, el acoger al otro es un acto que trasciende incluso a la hospitalidad domiciliaria. Y tiene que ver, ante todo, con esa capacidad de acogida que se llama comprensión. (Giannini, 1997, 341)

Si hospes alude al huésped y esto permite establecer una base hermenéutica para expresar el concepto de 'acogida', entonces, es posible inferir que aquél es acogido por otro que recibe y se recibe a sí mismo, en su afán por ser acogido por el huésped. Esto muestra al concepto de hospitalidad más allá de la comprensión de una acogida o alteridad unilateral. Es siempre recíproca. Lo mismo sucede entre un o una docente y sus estudiantes, desde el constructivismo como modelo educativo imperante junto con el sociocrítico estos últimos son cada vez más participativos de su proceso de aprendizaje, el o la docente fortalece más su rol de facilitador/a del conocimiento, pero también de las tecnologías al servicio de este fin.

Desde Ricoeur, infiero que la distinción fundamental, en el plano de la acogida, entre la alteridad entendida sólo en tanto tal y entre la alteridad emparejada con la ipseidad, radica en significar dicho concepto no desde una comparación, "[...] sí mismo semejante a otrosino de una implicación: sí mismo en cuanto...otro." (Ricoeur, 1996) Esta implicación es fundamental para entender el dualismo existencial, pues es proyección y reciprocidad al mismo tiempo. Esto también es hospitalidad, la implicación lo fundamenta y distancia de una alteridad del otro "con rostro".

Esto nos permite profundiza en la idea de internet como binomio entre individuo $\mathrm{y}$ comunidad.

\section{La era digital. Entre el individualismo y las comunidades virtuales}

En la actual sociedad del conocimiento y la información, entendida como era digital en el presente siglo, la brecha digital se debe trabajar 
desde dos categorías sumamente potentes, dos paradigmas propios de la sociedad contemporánea. Nos referimos al individualismo y a las comunidades virtuales.

Al igual que De Vega Alonso (2011), nos hacemos cargo de la definición que da Kuhn respecto a lo que entendemos por paradigma. Cómo se concibe el ser humano en esta era digital. Es sabido que en los campos de la economía y la política no hemos logrado superar esta supremacía del individuo, lo dicen los altos niveles de corrupción y de desigualdad existentes.

En cuanto al individualismo, nos interpela la propuesta de entenderlo como un paradigma:

Un individualismo que se mueve por egoísmo codicioso, un repliegue en uno mismo que cierra el sepulcro de la solidaridad y la fraternidad, una violencia que se manifiesta tanto en los brotes de terrorismo como en la trivialización de la delincuencia y la criminalidad, una democracia sin fervor cívico, un mercado que lo gobierna todo, derechos humanos desatendidos: el malestar cultural y ético también crece, hipertrofiado a la medida de un mundo híper en el que el individuo, cuanto más tiene, incluso cuando tiene demasiado, acaba preguntándose si tiene lo mejor. (Lipovetsky, 2010)

Ahora bien, también, es de amplio conocimiento la existencia de consolidadas comunidades virtuales (Cabero y Llorente, 2010), especialmente en el campo de la educación, dado que los nuevos entornos de enseñanza-aprendizaje mediados por TIC's así lo requieren. Esto se ha fortalecido dado el actual contexto de pandemia.

Estas comunidades, para que se logre el tránsito efectivo desde el paradigma del individualismo, deben contar con ciertas características y sortear ciertas dificultades.

Revisaremos a continuación una propuesta que caracteriza a las comunidades virtuales de aprendizaje (CVA):

- La interacción que se establece entre las personas que conforman la CVA se efectúa a través de máquinas, sean éstas fijas (ordenadores de sobremesa) o móviles (iPad, por ejemplo).
- Se caracterizan por el intercambio de información en diferentes formatos, y por la generación y construcción de conocimientos nuevos.

- Sus participantes comparten un lenguaje, pero no necesariamente unas creencias $y$ valores, aunque por supuesto, si lo comparten la CVA será más saludable.

- La interacción se realiza utilizando diferentes tipos de herramientas de comunicación, tanto sincrónica (chat) como asincrónicas (blog), y tanto textuales (wiki) como audiovisuales (videoconferencia).

- Y se realiza una comunicación multidireccional, que va de uno a uno, o de uno a todos. (Cabero y Llorente, 2010).

Dentro de las dificultades que tienen las CVA, sin lugar a duda que durante la pandemia se han reforzado y evidenciado ciertas desigualdades sociales que, a su vez, han incrementado la brecha digital en contextos educativos. Podemos mencionar las más comunes (sin orden de prioridad):

- Económicas (acceso a la adquisición o contratación de productos o servicios de conectividad)

- Geográficas (acceso equitativo independiente de la condición de urbanidad o ruralidad de la zona. Y acceso descentralizado independiente de la ubicación y si ésta es más aislada o no que otra)

- Generacionales (asociadas a la diferencia de acceso por uso y conocimientos propios de pertenecer a distintos rangos etarios y su respectiva diversidad de aprendizajes previos o experiencias respecto a las tecnologías digitales)

- Interculturales (referido a la diferencia existente en el acceso al uso de tecnologías digitales por parte de grupos o comunidades pertenecientes a pueblos o naciones indígenas y a grupos de personas migrantes con origen distinto al país de residencia)

- En ámbito de inclusión (asociadas a las desigualdades generadas como consecuencia de encontrarse en alguna situación de discapacidad y enfrentarse a limitaciones de plataformas que no consideran accesibilidad universal) 
- En ámbito de género (acceso diferenciado o con rasgos de exclusión por motivos culturales que incorporan un lenguaje no inclusivo o binario. 0 como consecuencia de una enseñanza con características patriarcales, donde las mujeres están asociadas a labores de cuidado del hogar o subalternas a los hombres, entendiendo como no prioritaria la alfabetización digital en igualdad de condiciones y de calidad).

$\mathrm{Si}$ podemos superar estas desigualdades, sin duda habremos logrado transitar de un paradigma del individualismo a uno de las comunidades (incluyendo las virtuales de aprendizaje)

A continuación, revisaremos algunas consideraciones respecto al concepto de brecha digital.

\section{Una comprensión sobre el concepto de brecha digital}

Tanto Rodríguez (2006) como Serrano y Martínez (2003), coinciden en que la llamada brecha digital considera al menos las siguientes características:

- Diferencia entre personas y sociedades o comunidades respecto a la utilización de tecnologías de la información, comunicación e informática.

- El uso/no uso de aparatos telefónicos, computacionales e internet.

- El desarrollo tecnológico está relacionado no sólo con el desarrollo económico, sino que también con el acceso a la educación, factores sociales, culturales, políticos, demográficos, etarios, de género, entre otros. Tal como describimos en párrafos anteriores.

Esto nos lleva a considerar que la brecha digital se mantendrá vigente durante un largo plazo de tiempo y en diversos países del mundo, ya que la desigualdad existente es muy difícil de resolver en el corto plazo, dada la escasa existencia de políticas públicas de Estado que sean robustas en inversión y en equidad.

Además, es relevante mencionar lo siguiente:

cuando se trata de definir el problema de la brecha es conveniente tener en cuenta que ésta se relaciona con las políticas públicas de acceso a la información y no debe confundirse con la simple disponibilidad o carencia de instrumentos tecnológicos. Cuando se comete ese error se intenta sustituir la política con la tecnología. (Rodríguez, 2006)

Esto es relevante para el desarrollo de políticas de innovación docente en la educación superior, especialmente en la formación inicial docente, ya que no basta con mejorar la infraestructura tecnológica ni el acceso de los estudiantes y docentes a las nuevas tecnologías de la información y comunicación como lo pueden ser las pizarras digitales o softwares especializados. Se requiere de claras definiciones de cómo lograr que todos y todas tengan cada vez un mejor acceso a la información y conectividad, para así lograr una mejor toma de decisiones.

De eso se trata la hospitalidad digital también, de saber tomar decisiones que permitan reducir la brecha digital, no sólo por acceso al uso de la red o herramientas virtuales, sino por acceso a información de calidad y, principalmente, para poder hacer cambios e implementar o diseñar herramientas digitales que otorguen sentido y significado al proceso de enseñanza $y$ aprendizaje mediado por tecnologías digitales.

Este fenómeno social está cruzado con el bajo nivel educativo de aquellos que además están en desventaja por las desigualdades descritas anteriormente.

Siguiendo lo trazado en páginas anteriores, establecemos una clara relación con el concepto de extranjero del que hablaba Ricoeur (2005), por ello la hospitalidad digital hace sentido en la actual era digital, ya que permite establecer parámetros de reflexión y comprensión respecto a la desventaja en la que se encuentran muchas personas y sociedades que son foráneas ante el acceso a la información y al uso de las tecnologías. Estamos llamados y llamadas a usar nuestra capacidad de reconocer y traducir la brecha digital.

La brecha digital no es otra cosa que el reflejo de la brecha social en el mundo digital. Si bien se debe considerar que el uso de las TIC para el desarrollo humano porta oportunidades para reducir la brecha social para individuales o comunidades, existen una serie de obstáculos a superar para que el uso de las TIC permita acercar esas oportunidades a las personas y a 
los grupos, dentro de los cuales la existencia de una infraestructura de conectividad es sólo el primero. No es suficiente ofrecer un acceso a las tecnologías para que las personas gratificadas con el uso puedan aprovechar las oportunidades de desarrollo humano; la educación y más específicamente una alfabetización digital e informacional tienen un papel central. Los componentes de las telecomunicaciones, de los equipos de computación y de los programas son requisitos previos y previsibles; sin embargo, los pilares verdaderos de las sociedades de la información centradas en el desarrollo humano (sociedades de los saberes compartidos) son la educación, la ética y la participación articuladas como un proceso sistémico. Mientras las personas que toman decisiones para políticas públicas o proyectos de TIC para el desarrollo no sean educadas para entender esas evidencias sufriremos de la brecha más peligrosa en términos de efectos: la brecha paradigmática. (Pimienta, 2008)

Esta cita de Pimienta nos parece sumamente decidora al momento de describir lo que entendemos por brecha digital, ya que da cuenta de la característica de reproducir la sociedad de la información en general y distingue claramente que lo importante no está en la infraestructura digital, sino en el uso que le damos y en la calidad de la información que se nos presenta y a la cual podemos y debemos acceder.

Aún más decidora es la relación que hace Alva de la Selva (2015), al hablar de la brecha digital como la nueva desigualdad, "en términos de las inequidades sociales en materia de acceso, uso y apropiación de las Tecnologías de la Información y la Comunicación (tic)". Ya la CEPAL lo aclara al indicar la necesidad de "superar la brecha digital, la cual refleja e incide en las diferencias económicas, sociales, culturales, educacionales, de salud y acceso al conocimiento, entre los países y dentro de ellos" (CEPAL, 2009, 57).

Las promesas de la sociedad del conocimiento no se cumplieron en esta creciente globalización, al menos en materia de la llamada brecha digital, ya que los niveles de desigualdad social, económica y cultural seguían creciendo. Al mismo tiempo se van constatando nuevas desigualdades, como las provocadas por los distintos niveles de inserción en redes globales o una llamada desigualdad por desconexión. Por ello es que las distintas instituciones educativas tuvieron que asignar recursos para becas de conectividad en su comunidad estudiantil y académica.

De hecho, al hablar de brecha digital como una nueva desigualdad, no podemos obviar lo dicho por García Canclini, respecto a que "los incluidos son quienes están conectados; sus otros son los excluidos, quienes ven rotos sus vínculos al quedarse sin trabajo, sin casa, sin conexión" (García Canclini, 2006: 73).

Esta desconexión y desigualdad nos interpela profundamente desde el concepto de hospitalidad digital que proponemos, ya que éste nos permite abordar la problemática desde raíces y constructos de un paradigma propio de la ética del siglo XXI, como lo es la ética de la comprensión (Morin, 1999) y si traducir es comprender como señalamos en apartados anteriores y traducir es hospedar, entonces la hospitalidad digital, también favorece la comprensión del contexto que propicia la creciente brecha digital en que vivimos, porque la desigualdad sigue siendo el gran mal de Latinoamérica, el Caribe y África, entre otras regiones.

Y una de las consecuencias más directas de la brecha digital es el analfabetismo digital.

Acá unas definiciones interesantes:

entendemos un proceso continuo que va más allá de la simple adquisición de la capacidad para leer y escribir, comprender elementalmente un concepto o manejar rudimentariamente un instrumento $y$ por "digital" entendemos todas las manifestaciones culturales y sociales que se originan, apoyan o transmiten con el recurso de las tecnologías de la información y el conocimiento. Tenemos, por tanto, que "alfabetización digital" podría ser el proceso de adquisición de los conocimientos necesarios para conocer y utilizar adecuadamente las infotecnologías y poder responder críticamente a los estímulos y exigencias de un entorno informacional cada vez más complejo, con variedad y multiplicidad de fuentes, medios de comunicación y servicios. (Casado, 2006)

Entonces, entendemos por analfabetismo digital, el estado de desconocimiento respecto a la sociedad de la información, era digital y al uso de tecnologías de la información y el 
conocimiento. Por ello es clave generar en la actualidad instancias de capacitación y formación en esta materia, para con ello ir acortando la brecha digital existente.

A modo de ejemplo, Corea del Sur es:

uno de los países que tiene uno de los crecimientos más acelerados en acceso a Internet, el mayor problema es la falta de habilidades para hacer usos efectivos de Internet. De acuerdo con la Oficina Nacional de Estadística de Corea del Sur, el 60 por ciento de los habitantes de ese país eran analfabetos computacionales en 1997. En otra encuesta que se realizó en 2000, se encontró que el 46 por ciento de los coreanos carecen de conocimientos sobre computadoras y de cómo navegar en la red. (Rodríguez, 2006)

Este ejemplo nos ayuda a señalar que no importa solamente aumentar el acceso a la tecnología, sino que es primordial educar digitalmente, alfabetizar en esta línea. Para ello, la hospitalidad digital considera la apertura a la alfabetización digital en nuestras instituciones educativas, pero también en espacios informales y no formales de la educación.

Por ello relacionamos hospitalidad digital con derechos humanos, dado el horizonte utópico, ético y jurídico, que busca reducir las desigualdades y garantizar, en este caso, el acceso igualitario a la información, conectividad y uso de tecnologías digitales para la educación.

\section{Tecnología digital y derechos humanos}

Es importante establecer la relación existente entre las tecnologías digitales y los derechos. Es relevante considerar el acceso a las tecnologías de la información y comunicación y su valor en el desarrollo de la desigualdad que conlleva a la llamada brecha digital, esto forma parte de un amplio debate sobre los posibles beneficios $y$ riesgos de la era digital.

Esto se debe a que las TIC son, en sí mismas, un dinamizador clave de la globalización: el nivel y el ritmo de las corrientes mundiales en activos materiales e inmateriales se han incrementado de manera espectacular por la gran capacidad de conectar personas a las redes, atravesando fronteras geográficas, a bajo costo. Esta relación entre las TIC y la globalización hace que las TIC requieran de intervenciones críticas en las políticas de desarrollo y cambio social. (Del Río Sánchez, 2009)

Aunque las TIC no son el objetivo en sí mismo, ya que sí lo es el acceso a la información -que las TIC facilitan-, dado que:

ayuda/puede ayudar a la gente a identificar y evaluar oportunidades de crecimiento y desarrollo y a mejorar sus vidas y las de sus familiares y comunidades. El acceso a la información facilita la participación en la sociedad, en la economía, en el gobierno y en los mismos procesos de desarrollo. (Del Río Sánchez, 2009)

Incluso, se puede establecer lo siguiente:

las TIC tienen, por un lado, el valor de la información para el ejercicio de derechos, como por ejemplo el valor que pueda tener la información sobre sus derechos para la población inmigrada, o bien el valor que puede tener la disposición de información (accesible y útil) sobre opciones políticas, mecanismos de votación o derechos laborales. (Acevedo, 2004).

Así también, la comunicación tiene el valor que permite ejercer, por ejemplo, el derecho a la participación en la vida política o el derecho a la libertad de expresión (Acevedo, 2004).

En el 2004, la Agencia Suiza de Cooperación al Desarrollo desarrolló un esquema conceptual de las TIC para el desarrollo donde se establecen metas y dimensiones clave de las TIC para el desarrollo (Del Río Sánchez, 2009).

Figura 1. Metas principales y dimensiones clave de las TIC para el desarrollo

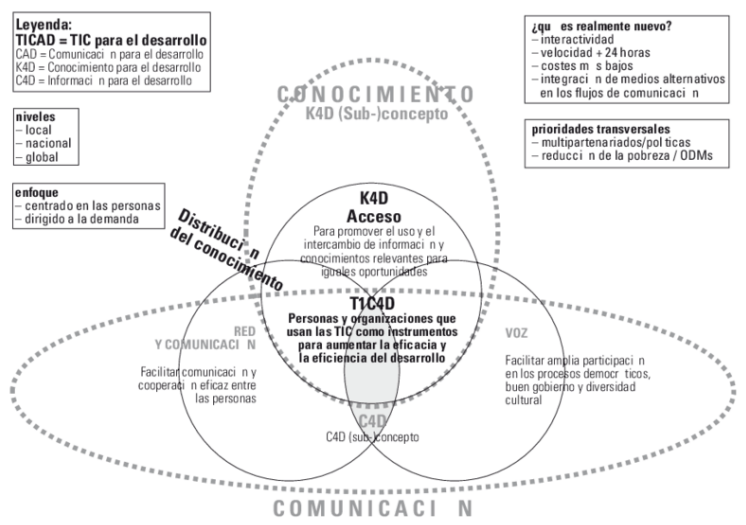

Fuente: Del Río, 2009. 
Por ello volvemos a aludir a Kofi Annan, quien como secretario general de las Naciones Unidas declaró en el Día Mundial de las Telecomunicaciones de 2003 que "...millones de personas de los países más pobres siguen estando excluidas del derecho a comunicarse que, cada vez más, se considera un derecho humano fundamental".

La era digital y el uso de tecnologías digitales, además de la llamada brecha digital abren un escenario para la defensa, promoción o, incluso, para la vulneración de los derechos humanos. En la Declaración del Milenio del año 2000 los países participantes acordaron velar por que todos puedan aprovechar los beneficios de las nuevas tecnologías, en particular de las tecnologías de la información y de las comunicaciones (Del Río Sánchez, 2009).

Aun cuando hablar de generaciones de derechos humanos está quedando obsoleto, dada la comprensible discusión en torno a la continuidad histórica de los mismos, en vez de su segmentación temporal, cuando se estableció que la cuarta generación de derechos humanos contemplaba los derechos a la información, la comunicación, el ciberespacio y el conocimiento, también se hablaba del contexto de la sociedad de la información y de la era digital.

Ya lo menciona Del Río Sánchez (2009), de que en 1996, la UNESCO publicó un documento de orientación titulado "La UNESCO y la sociedad de información para todos", en el cual se expone la obligación de impulsar la cooperación internacional en el terreno de la comunicación, la información y la informática, con objeto de reducir la desigualdad imperante (brecha digital para efecto de nuestro artículo) entre los países desarrollados y los que se encuentran en vías de desarrollo; colocando un especial énfasis en el uso de las TIC al servicio del desarrollo, de la democracia y de la paz.

También, la Asamblea General de Naciones Unidas el 27 de junio de 2016 incluye nuevamente el tema "Promoción, protección y disfrute de los derechos humanos en Internet" por parte de su Consejo de Derechos Humanos indicando, entre otros puntos, lo siguiente:
Afirma también la importancia de que se aplique un enfoque basado en los derechos humanos para facilitar y ampliar el acceso a Internet y solicita a todos los Estados que hagan lo posible por cerrar las múltiples formas de la brecha digital. (ONU, 2016)

Acá es donde la educación en derechos humanos juega un rol fundamental y desde donde podemos entender el cruce con el concepto de hospitalidad digital que definiremos a continuación.

\section{Concepto de hospitalidad digital}

Ahora podemos definir este concepto. La hospitalidad digital busca ser considerada como una de las llamadas competencias para el siglo XXI y se entiende como una aproximación reflexiva basad en la hermenéutica y como una propuesta filosófico educativa que permite abordar las diversas categorías que surgen en el contexto de una avanzada era digital y disminuir la actual brecha digital y uno de sus principales efectos, el analfabetismo digital, el cual trae otras consecuencias en la sociedad, tales como las vivida dramáticamente en contexto de pandemia.

También, la hospitalidad digital se entenderá como aquella disposición del ser humano para comprender y hacerse cargo de una era digital que denota el amplio desarrollo de la sociedad del conocimiento y la información que a nuestra generación le ha tocado experimentar. Esta comprensión tiene darse, al menos, desde una reflexión ética, respetuosa de la diversidad y con un enfoque de derechos humanos.

Así se irá construyendo este concepto, el cual se fortalecerá en la medida que logremos vincularlo con el desafío que supone educar con sentido y por medio de las tecnologías digitales que hoy en día se utilizan.

Por último, la hospitalidad digital, esperamos se transforme en una alternativa para que los y las docentes se permitan el espacio de la innovación educativa para eliminar las barreras de la desigualdad propias de dicha brecha digital. 


\section{Referencias}

Acevedo, M. (2004). Las TIC en las políticas de cooperación al desarrollo: hacia una nueva cooperación en la Sociedad en Red. En Cuadernos Internacionales de Tecnología para el Desarrollo Humano, 2.

Alva de la Selva, A. (2015). Los nuevos rostros de la desigualdad en el siglo XXI: la brecha digital. Revista Mexicana de Ciencias Políticas y Sociales. DOI: 10.1016/S0185-1918(15)72138-0

Annan, K. (2003) Discurso para CEPAL. Recuperado de: https://www.cepal.org/cgibin/getProd.asp?xml=/prensa/noticias/comunicados/6/12266/P12266.xml\&xsl=/prensa/tpl/p6 f.xsl\&base=/prensa/tpl/top-bottom.xsl

Benveniste, E. (1983). El vocabulario de las Instituciones Indoeuropeas. España: Taurus

Cabero, J. y Llorente, M. (2010). Comunidades virtuales para el aprendizaje. España. En Edutec. Revista Electrónica de Tecnología Educativa, 34.

Capurro, R. (2010) La hermenéutica frente al desafío de la técnica digital. Brasil: en Liinc em Revista, V.6, $\mathrm{N}^{\circ} 2$. Recuperado de: http://ibict.br/liine

Casado, R. (2006). Claves de la alfabetización digital. Chile: Fundación Telefónica.

Cassany, D. (2002). La alfabetización digital. Costa Rica: ALFAL

CEPAL. (2009) La sociedad de la información en América Latina y el Caribe, CEPAL-IDRC-Europe Aid, Santiago de Chile.

Cervera, Hernández y Adsuar. (2005). El Ensayo como género literario. España: Universidad de Murcia. Recuperado de: file://C:/Users/UCSH/Downloads/971-21-1131-1-10-20131208.pdf

De Vega, E. (2011). El individualismo: ¿paradigma del hombre contemporáneo? España: UNED. Recuperado de: http://e-spacio.uned.es/fez/eserv/tesisuned:Filosofia-Evega/Documento.pdf

Del Río Sánchez, O. (2009) TIC, derechos humanos y desarrollo: nuevos escenarios de la comunicación social. En Anàlisi 38. España.

Díez Fischer, F. (2010). La hospitalidad lingüística ¿A quién hospeda, quien traduce? En Encuentro Nacional de Fenomenología y Hermenéutica (28-30 septiembre).

Ferraris, M. (2005) Historia de la hermenéutica. México: Siglo XXI

García Canclini, N. (2006) Desiguales y desconectados, Gedisa, Barcelona.

Giannini, H. (1997) Hospitalidad y tolerancia (o de la tolerancia), Chile: En Estudios Públicos, 66, p. 341.

Grondin, J. (1999) Introducción a la hermenéutica filosófica. España: Herder.

Lipovetsky, G. \& Serroy, J. (2010) La cultura-mundo.: Respuesta a una sociedad desorientada. Anagrama.

Morin, E. (1999) Los Siete saberes necesarios para la educación del futuro. UNESCO. Recuperado de: https://unesdoc.unesco.org/ark:/48223/pf0000117740_spa

ONU. (2016) Recuperado de: https://ap.ohchr.org/documents/S/HRC/d_res_dec/A_HRC_32_L20.pdf

Pérez, A. (2013). La era digital: nuevos desafíos educativos. En Revista Electrónica Sinéctica, 40, 47-72.

Recuperado de: http://www.redalyc.org/articulo.oa?id=99827467010

Pimienta, D. (2007). Brecha digital, brecha social, brecha paradigmática. Santo Domingo: Funredes.

Rebollo, M. García, R. Barragán, R. Buzón, O. y Vega, L. (2008) Las emociones en el aprendizaje online. España: en RELIEVE, v. 14, n. 1, p. 1-23. Recuperado de: http://www.uv.es/RELIEVE/v14n1/RELIEVEv14n1_2.htm

Ricoeur, P. (1993). Amor y Justicia. España: Caparrós Editores.

- (1996). Sí mismo como otro. España: Siglo XXI

- (1999). Historia y narratividad. España: Paidos

- (2005). Sobre la traducción. España: Paidos

Rodríguez, A. (2006). La brecha digital y sus determinantes. México: UNAM

Serrano, A. y Martínez, E. (2003). La brecha digital: mitos y realidades. México: Universidad Autónoma de Baja California.

Steiner, G. (1977). Después de babel. Aspectos sobre el lenguaje y la traducción. México. FCE. 